\title{
USO DO CONTRASTE ORAL NEGATIVO EM EXAMES DE COLANGIOGRAFIA POR RESSONÂNCIA MAGNÉTICA*
}

\author{
Mário de Melo Galvão Filho ${ }^{1}$, Giuseppe D’lppolito ${ }^{2}$, Maria Lucia Borri ${ }^{2}$, Angela M. Borri \\ Wolosker ${ }^{1}$
}

Resumo OBJETIVO: Realizamos estudo prospectivo das vias biliares e pancreáticas através de colangiografia por ressonância magnética, com a utilização de meio de contraste oral negativo. Os nossos objetivos foram verificar se este novo meio de contraste melhora a visualização das vias biliar e pancreática, além de identificar a freqüência de efeitos colaterais ao contraste e sua aceitação pelo paciente. MATERIAL E MÉTODO: Quinze voluntários (oito homens e sete mulheres) com idades variando entre 18 e 54 anos (média de 29 anos), sem queixas ou cirurgias abdominais, foram submetidos a colangiografia por ressonância magnética. Foram realizadas duas seqüências colangiográficas em apnéia, antes e cinco minutos após a ingestão de $300 \mathrm{ml}$ de contraste oral negativo. Os exames foram realizados em equipamento operando a 1,0 T. RESULTADOS: Setenta e três por cento dos voluntários consideraram o gosto ruim ou muito ruim, sugerindo uma aceitação discutível; $27 \%$ dos voluntários apresentaram náuseas; $20 \%$, cólicas; $14 \%$, azia ou parestesia labial; e 7\%, diarréia. A visualização da via biliar extra-hepática foi considerada melhor após o contraste oral negativo em $9 / 15$ voluntários $(60 \%)$ e do ducto pancreático principal em todos os cinco em que havia interposição de alças. CONCLUSÃO: 0 contraste oral negativo melhora a visualização dos ductos hepatocolédoco e pancreático principal em exames de colangiografia por ressonância magnética, apesar da baixa aceitação e dos seus efeitos colaterais.

Unitermos: Ressonância magnética. Vias biliares. Duto pancreático. Meio de contraste.

\begin{abstract}
Use of oral negative contrast agent in magnetic resonance cholangiopancreatography examinations. OBJECTIVE: The aim of this prospective study was to investigate the feasibility of using a negative oral contrast agent to null the bowel signal during magnetic resonance cholangiopancreatography. MATERIAL AND METHOD: Fifteen healthy volunteers with no previous history of pancreaticobiliary disease or surgery were imaged with a single-shot fast spin-echo pulse sequence, using a magnetic resonance imaging system operating at 1.0 T. Data acquisition was started before and after administration of oral contrast agent. Using the magnetic resonance images obtained before and after administration of oral contrast agent, the common bile duct, pancreatic duct and papilla were assessed and graded by two radiologists. The frequency of adverse effects and the tolerance of the contrast were also evaluated. RESULTS: All patients that found negative oral contrast agent unpleasant (73\% of the cases) tolerated well the oral contrast agent. Adverse effects as abdominal pain and diarrhea were noted in $30 \%$ of the patients. In all patients the high signal intensity from the intestinal fluid was completely suppressed. The depictions of the common hepatic duct were slightly improved, whereas the depictions of the choledochus, papilla and pancreatic duct were markedly improved by the negative oral contrast agent administration. CONCLUSION: Negative oral magnetic resonance contrast agent can be an effective and safe contrast media in eliminating signal intensity of the gastrointestinal tract, thus improving the depiction of the biliary system in magnetic resonance cholangiopancreatography.

Key words: Magnetic resonance imaging. Biliary system. Pancreatic duct. Contrast media.
\end{abstract}

\footnotetext{
* Trabalho realizado no Serviço de Ressonância Magnética do Complexo Hospitalar São Luiz, São Paulo, SP.

1. Médicos do Serviço de Tomografia Computadorizada, Ressonância Magnética e Ultra-Sonografia do Com plexo Hospitalar São Luiz, Doutores em Medicina pela Universidade Federal de São Paulo/Escola Paulista de Medicina (Unifesp/EPM).

2. Médicos do Serviço de Tomografia Computadorizada, Ressonância Magnética e Ultra-Sonografia do Complexo Hospitalar São Luiz, Doutores em Medicina e Professores Adjuntos da Unifesp/EPM.

Endereço para correspondência: Dr. Mário de Melo Galvão Filho. Rua Jacutinga, 225, $5^{\circ}$ andar, Moema. São Paulo, SP, 04515-003.E-mail: mgalvao@uol.com.br

Recebido para publicação em 10/1/2002. Aceito, após revisão, em 20/6/2002.
}

\section{INTRODUÇÃO}

Os exames de colangiografia por ressonância magnética (colangio-RM), por serem rápidos e não-invasivos, têm ocupado, progressivamente, o espaço da colangiopancreatografia retrógrada endoscópica (CPRE) com fins diagnósticos. Suas indicações são bastante precisas e difundidas. A colangiopancreato-RM tem sido utilizada na avaliação de pacientes com suspeita de coledocolitíase, previamente a colecistectomias laparoscópicas, esclarecimento diagnóstico de dilatação das vias biliares, com ultra-som (US) inconclusivo ou divergente do quadro clínico e em estudos funcionais de pacientes com pancreatite crônica e disfunção do esfíncter de Oddi ${ }^{(1)}$.

As seqüências utilizadas são fortemente ponderadas em T2, com tempos de eco muito longos determinando um intenso sinal dos fluidos estáticos ou de fluxo lento, como a bile, ocorrendo também uma redução de sinal dos tecidos adjacentes ${ }^{(2)}$. Freqüentemente, o sinal de secreções gastroduodenais sobrepõe-se ao sinal das vias 
biliar e pancreática, dificultando suas avaliações nos exames de colangio- $\mathrm{RM}^{(2)}$.

Recentemente, vários contrastes orais têm sido desenvolvidos e utilizados para aperfeiçoar o exame de RM do abdome, com o intuito de delinear o tubo digestivo; esses contrastes são denominados negativos ou positivos, em função do aspecto que conferem às alças intestinais ${ }^{(3)}$. Com o objetivo de reduzir as limitações ligadas à sobreposição de intestino com líquido, alguns meios de contraste oral capazes de anular o sinal no interior do tubo digestivo nas imagens ponderadas em T2 são utilizados e denominados meios de contraste negativo. Estes contrastes consistem em uma suspensão de cristais de óxido de ferro, superparamagnéticos, revestidos de silicone, e apresentam elevada suscetibilidade magnética ${ }^{(3)}$.

Os fabricantes desses meios de contraste preconizam a administração de volume elevado (cerca de $900 \mathrm{ml}$ ), e talvez por esta razão e por apresentarem sabor pouco agradável, além de aumentar o custo do exame, não têm sido utilizados sistematicamente $^{(3)}$. Porém, temos notado, com certa freqüência, em exames de colangio-RM, que o resíduo alimentar e suco gástrico têm dificultado a avaliação do colédoco distal, região da papila e ducto pancreático, mesmo quando o paciente mantém o jejum preconizado. Os contrastes negativos, nestes casos, poderiam facilitar a interpretação do estudo colangiográfico por RM. Além disso, novos trabalhos referendam o uso de contrastes negativos para auxiliar a interpretação de exames de colangio-RM realizados para a avaliação da função pancreática, mediante estímulo com secretina ${ }^{(4)}$.
A literatura internacional ainda é escassa no que concerne à real utilidade desses contrastes. No Brasil, o contraste oral negativo para RM só foi disponibilizado no início de 2000, tendo-se pouca experiência com o seu uso, motivos estes que nos estimularam a realizar este trabalho.

Os nossos objetivos foram avaliar a aceitação do meio de contraste oral negativo para RM (Lumirem ${ }^{\circledR}$, Guerbert), estabelecer a sua utilidade na visualização das vias biliar e pancreática e verificar a freqüência dos seus efeitos colaterais.

\section{MATERIAL E MÉTODO}

Realizamos trabalho prospectivo, entre outubro de 2000 e março de 2001, utilizando seqüências colangiográficas por RM em 15 voluntários, sendo oito do sexo masculino e sete do sexo feminino, com idades variando entre 18 e 54 anos (média de 29 anos), sem qualquer queixa ou antecedente cirúrgico das vias biliar e pancreática. Os exames foram realizados em equipamento de RM modelo Gyroscan NT10 (Philips), operando a 1,0 T, com gradiente de $15 \mathrm{mT}$ e com bobina de corpo.

Os voluntários se apresentavam nas primeiras horas da manhã, com oito horas de jejum. Foram realizadas duas seqüências ponderadas em T2 (TR: 10.800; TE: 900; FA: $90^{\circ}$; N: 12 cortes, em apnéia; espessura: $5 \mathrm{~mm}$; plano coronal; $9 \mathrm{~s}$ ) antes e cinco minutos após a ingestão de $300 \mathrm{ml} \mathrm{de}$ contraste oral negativo.

Dois radiologistas em consenso avaliaram a visualização do hepatocolédoco, ducto pancreático principal e papila, nas duas seqüências (pré e pós-ingestão do contraste). Procurou-se determinar, independentemente da nitidez da visualização das vias biliar extra-hepática e pancreática, se havia sobreposição entre estas estruturas e porções do estômago ou duodeno.

Para se avaliar o grau de aceitação do contraste, os voluntários responderam subjetivamente se o gosto do produto era muito ruim, ruim, indiferente, bom ou muito bom. No dia seguinte ao exame os voluntários foram questionados sobre efeitos colaterais, como náusea, azia, cólicas, diarréia ou parestesias perilabiais.

Utilizamos o teste de McNemar para verificar a existência de discordância entre as seqüências pré e pós-contraste quanto à sobreposição do tubo digestivo sobre as vias biliar e pancreática. Calculamos também as freqüências de efeitos colaterais e a aceitação do produto, por intermédio de questionário apropriado.

\section{RESULTADOS}

\section{Visualização das vias biliar e pancreática}

Ao avaliarmos a visualização do ducto colédoco, região da papila de Vater e ducto pancreático comum, em função da sobreposição de resíduo no estômago e duodeno, antes e após a ingestão do contraste, encontramos os seguintes resultados:

Em 6/15 voluntários (40\%) houve boa visualização do colédoco, seja na fase pré como na pós-contraste. Em 9/15 voluntários $(60 \%)$ que apresentavam sobreposição do duodeno ou estômago sobre o colédoco antes da ingestão do meio de contaste oral, esta sobreposição não ocorreu após o contraste (Figura 1). Segundo o teste de

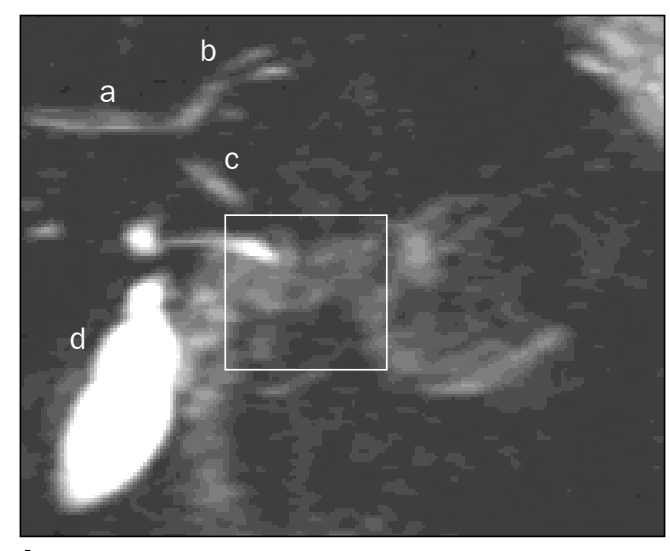

A

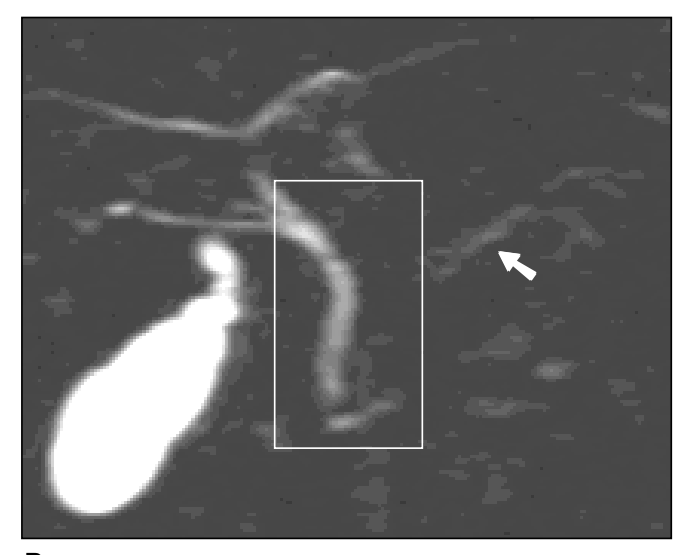

B
Figura 1. A: Colangio-RM sem contraste oral negativo, observando-se sobreposição do hepatocolédoco pelo arco duodenal ( $\square$ ). Não há sobreposição de via biliar hepática direita (a), esquerda (b), hepatocolédoco proximal (c) e vesícula biliar (d). B: Colangio-RM após utilização de contraste oral negativo, observando-se a ausência de sinal, tanto no estômago (seta) quanto no duodeno, com visualização do hepatocolédoco (D). 
McNemar, podemos afirmar que existe uma discordância não-casual entre a utilização ou não do meio de contraste oral negativo, com $\mathrm{p}=0,002 *$ (Tabela 1 ), para a visualização do colédoco.

Em relação ao ducto pancreático comum, pudemos observar que 5/15 voluntários $(33,33 \%)$ que mostravam sobreposição com o estômago não a tiveram após o contraste negativo (Figura 2; Tabela 2). Em apenas um voluntário notamos que a sobreposição gástrica sobre o ducto pancreático não desapareceu após a ingestão do contraste negativo. Pudemos constatar, estatisticamente, que a melhora nos cinco casos não ocorreu por acaso e pôde ser creditada, assim como a melhor visualização do colédoco, ao meio de contraste oral.

A visualização da região da papila não pôde ser estatisticamente avaliada (Tabela $3)$, pois $11 / 15$ voluntários $(73,33 \%)$ não apresentavam sobreposição duodenal já na fase pré-contraste (Figura 3). No entanto, nos quatro casos em que o duodeno com líquido impedia a adequada visualização
Tabela 1 Visualização do colédoco antes e após o uso do meio de contraste oral negativo.

\begin{tabular}{|l|c|c|c|}
\hline Pós & Com sobreposição & Sem sobreposição & Total \\
\hline Com sobreposição & 0 & 0 & 0 \\
Sem sobreposição & 9 & 6 & 15 \\
\hline Total & 9 & 6 & 15 \\
\hline
\end{tabular}

$P=0,002 *$ ou $0,2 \% *$

Tabela 2 Visualização do ducto pancreático comum antes e após o uso do meio de contraste oral negativo.

\begin{tabular}{|l|c|c|c|}
\hline Pós & Com sobreposição & Sem sobreposição & Total \\
\hline Com sobreposição & 1 & 0 & 1 \\
Sem sobreposição & 5 & 9 & 14 \\
\hline Total & 6 & 9 & 15 \\
\hline
\end{tabular}

$P=0,031^{*}$ ou $3,1 \% *$.

Tabela 3 Visualização da papila antes e após o uso do meio de contraste oral negativo.

\begin{tabular}{|l|c|c|c|}
\hline Pós & Com sobreposição & Sem sobreposição & Total \\
\hline Com sobreposição & 1 & 0 & 1 \\
Sem sobreposição & 3 & 11 & 14 \\
\hline Total & 4 & 11 & 15 \\
\hline
\end{tabular}

Figura 2. A: Colangio-RM sem contraste oral negativo, notandose sobreposição do estômago sobre o sinal do ducto pancreático (setas). B: Colangio-RM com contraste oral negativo, onde se observa o desaparecimento da sobreposição (setas).

Figura 3. Colangio-RM sem (A) e com (B) contraste oral negativo. A região da papila, por sua situação anatômica, não apresenta sobreposição já no pré-contraste $(\square)$. ( $(E$, estômago).

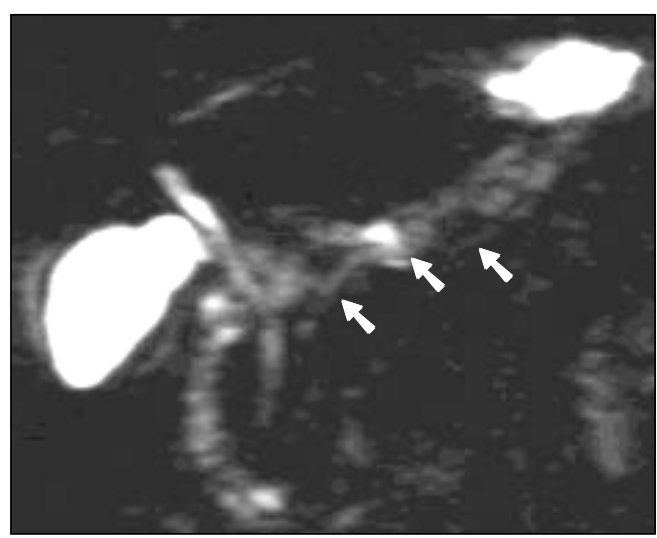

A

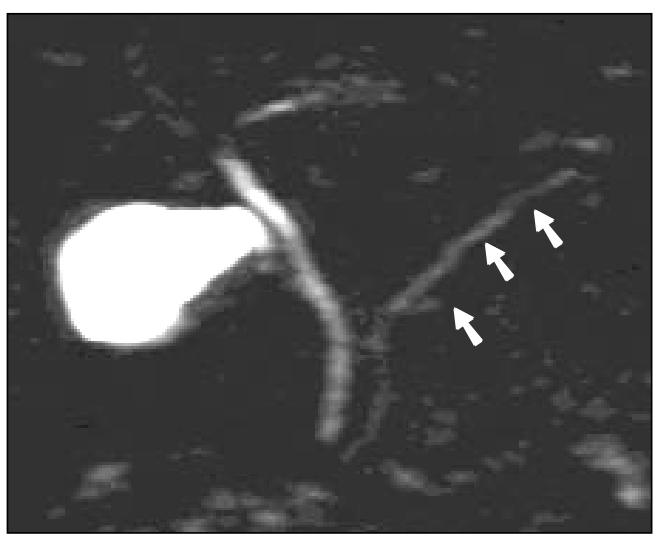

B

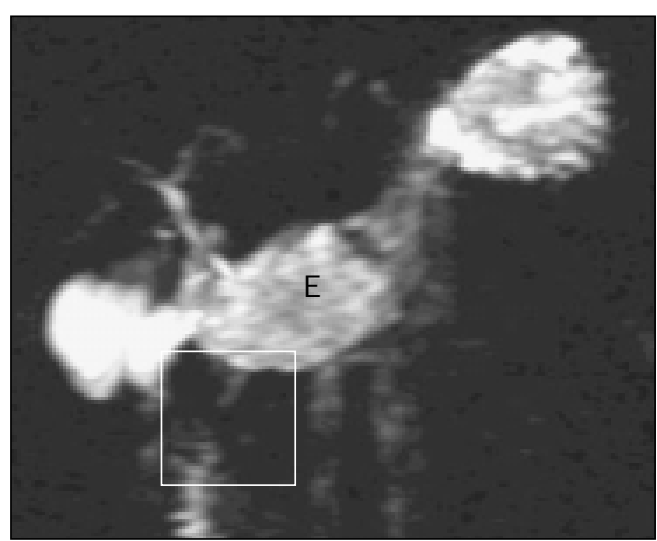

A

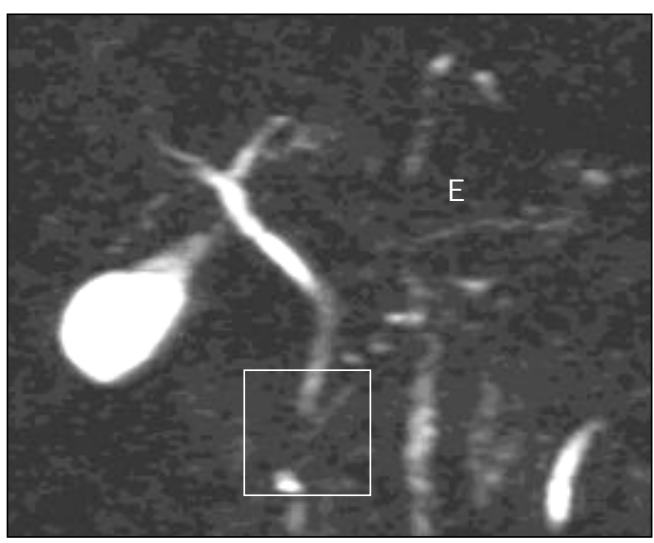

B 
da papila, o uso do contraste oral negativo permitiu uma melhor identificação desta região anatômica.

É importante salientarmos que em nenhum voluntário observamos pior visualização das estruturas em estudo após o uso do meio de contraste, e que dos 15 voluntários, dez apresentaram algum grau de melhora, sendo que em sete destes esta melhora foi considerada significativa.

\section{Aceitação do contraste}

A maioria dos voluntários (11/15; 73\%) considerou o gosto do contraste como ruim ou muito ruim, dois (14\%) o consideraram indiferente e outros dois (14\%) o consideraram bom (Tabela 4).

Tabela 4 Aceitação dos voluntários quanto ao gosto do meio de contraste, em números absolutos e porcentuais.

\begin{tabular}{|l|c|c|}
\hline \multicolumn{1}{|c|}{ Gosto } & $\begin{array}{c}\text { Número de } \\
\text { voluntários }\end{array}$ & Porcentual \\
\hline Muito ruim & 6 & $40 \%$ \\
Ruim & 5 & $33 \%$ \\
Indiferente & 2 & $14 \%$ \\
Bom & 2 & $14 \%$ \\
\hline Total & 15 & $100 \%$ \\
\hline
\end{tabular}

\section{Efeitos colaterais}

Os efeitos colaterais não foram infreqüentes, principalmente quanto à náusea. Em quatro casos ela se apresentou imediatamente após o início da ingestão, representando $27 \%$ dos voluntários. Nenhum voluntário, no entanto, apresentou vômitos. Três dos voluntários apresentaram cólicas consideradas brandas nas 24 horas seguintes, totalizando $20 \%$. Efeitos colaterais como azia, adormecimento da língua e diarréia foram experimentados em apenas um ou dois casos, porém considerados bastante desagradáveis (Tabela 5).

\section{DISCUSSÃO}

A colangio-RM tornou-se uma ferramenta importante para o estudo das vias biliar e pancreática, por ser rápida e nãoinvasiva. As reconstruções em 3D permitem avaliação semelhante à obtida pela CPRE e, principalmente, sem a necessidade de meio de contraste iodado ${ }^{(1)}$. Apesar de a colangio-RM ter ocupado o espa-

Tabela 5 Efeitos colaterais ao meio de contraste, em números absolutos e porcentuais.

\begin{tabular}{|l|c|c|}
\hline \multicolumn{1}{|c|}{ Efeito colateral } & Número de voluntários & Porcentagem \\
\hline Náusea & 4 & $27 \%$ \\
Cólicas & 3 & $20 \%$ \\
Azia & 2 & $14 \%$ \\
Diarréia & 1 & $7 \&$ \\
Adormecimento da língua & 1 & $7 \%$ \\
\hline
\end{tabular}

ço da CPRE diagnóstica, ainda apresenta resolução espacial inferior e algumas limitações ligadas não somente àquelas inerentes à RM, mas também a artefatos de sobreposição e ao fato de não permitir identificar claramente a permeabilidade das vias biliar e pancreática.

Assim como nós, outros autores apontaram alguns problemas e dificuldades de interpretação inerentes à sobreposição de sinal entre vias biliar e pancreática com os fluidos no interior do tubo digestório. $\mathrm{Na}$ verdade, estes problemas já haviam sido detectados anteriormente, mas não estudados adequadamente. Em 1987, Hahn et $a l .{ }^{(5)}$ foram os primeiros a estudar partículas ferrosas como meio de contraste para o uso no tubo digestório, em exames de colangio-RM. O principal objetivo dos autores foi facilitar a diferenciação entre alças intestinais, eventuais lesões intracavitárias e até mesmo distinguir melhor alguns órgãos parenquimatosos. Lonnemark et $a l .{ }^{(6)}$ também testaram meios de contraste oral negativo. Tratava-se de partículas superparamagnéticas não-biodegradáveis de óxido ferroso e magnetita envolvidas por um polímero carreador. Neste estudo, apesar da anulação do sinal proveniente do estômago e duodeno, perceberam a existência de alguns efeitos colaterais, como náusea e vômitos. Em outro trabalho(7), esses mesmos autores procuraram estudar o comportamento de sinal em concentrações diferentes deste contraste, ingeridas por 25 pacientes, que não referiram qualquer reação adversa ou efeito colateral. Diferentemente de outros trabalhos, atribuíram a ausência de efeitos colaterais à composição não biodegradável e à baixa concentração de cristais no meio de contraste utilizado.

Em 1993, Bach-Gansmo et al. ${ }^{(\mathbf{8})}$ submeteram 40 pacientes subseqüentes, sem patologia específica do abdome, a exame de RM. Os dez primeiros receberam con- centrações diferentes de contraste, com alta e baixa viscosidade, para escolha daquele que melhor se distribuiria pelo tubo digestório. A escolha recaiu sobre o de baixa viscosidade. Os pacientes foram preparados com meio de contraste oral negativo composto por micropartículas de óxido ferroso associadas à magnetita e recobertas por um polímero que as tornava não-biodegradáveis. Os autores tinham como objetivo estudar reações adversas, a distribuição do contraste pelo intestino e seu benefício ou não quanto à melhora da eficácia diagnóstica. Em seus resultados, observaram apenas uma reação adversa, caracterizada por vômito imediatamente após a ingestão do meio de contraste oral negativo. Trinta e três pacientes referiram indiferença em relação ao sabor e apenas dois relataram que nunca mais o utilizariam em hipótese nenhuma.

Os nossos resultados são bastante diferentes dos referidos na literatura, apesar do principal componente do Lumirem ${ }^{\circledR}$ ser basicamente igual ao dos contrastes utilizados nos trabalhos revisados (óxido ferroso revestido por silicone, porém sem a presença da magnetita). No entanto, são utilizados outros componentes na elaboração do Lumirem ${ }^{\circledR}$, não referidos na constituição dos contrastes utilizados por outros autores, tais como carbometilcelulose para aumento da viscosidade, corante, aroma de laranja e sacarina, entre outras substâncias.

Os nossos resultados demonstram baixa tolerância dos voluntários ao meio de contraste, traduzida por maior freqüência de efeitos colaterais. Esta diferença pode ser atribuída ao fato de não diluírmos o contraste, apesar de utilizarmos um volume menor que o preconizado. A utilização de corantes e aromatizantes pode gerar um efeito contrário ao objetivo esperado, de melhorar o aspecto visual e o paladar. Além disso, questionamos os pacientes 
sobre possíveis efeitos colaterais até 24 horas após a administração, justamente o período em que o número absoluto de efeitos indesejáveis aumenta, consideravelmente, em virtude de cólicas e diarréias. É importante salientarmos que a própria bula do meio de contraste adverte sobre esta possível ocorrência em até 24 horas após a ingestão. Em contrapartida, os trabalhos pesquisados, com exceção do de BachGansmo et al. ${ }^{\mathbf{( 8 )}}$, não fizeram tal questionamento, e desta maneira estariam subestimando estas ocorrências. E, por último, a diluição do meio de contraste com leite ou água ${ }^{\mathbf{( 7 , 8 )}}$ pode tê-lo tornado mais agradável, evitando principalmente as náuseas.

Também foi proposta uma mistura de água-leite e o meio de contraste negativo, como a melhor forma de homogeneizar a sua distribuição pelas alças intestinais. Apesar de uma incidência baixa de náuseas, $25 \%$ dos pacientes apresentaram diarréia, número próximo aos nossos para este efeito colateral.

Nossos pacientes também apresentaram um número considerável de cólicas e azia $(5 / 15 ; 34 \%)$, que podem estar relacionadas à elevada concentração de ferro e outras substâncias presentes no Lumirem ${ }^{\circledR}$. Em nenhum outro trabalho a parestesia labial, referida na bula do Lumirem $^{\circledR}$, foi citada, sendo que um (7\%) dos nossos voluntários a apresentou.

A aceitação do meio de contraste oral negativo pelos nossos voluntários foi baixa, uma vez que $77 \%$ deles acharam o gosto ruim ou muito ruim. Talvez a mistura/ diluição do produto em leite, água ou ambos, como proposta por outros autores ${ }^{(\mathbf{1}}$, ${ }^{7,8)}$, possa melhorar a aceitação do meio de contraste.

Em relação ao nosso principal objetivo, ou seja, medir a melhora na visualização das vias biliar e pancreática, decorrente da anulação do sinal proveniente dos fluidos no interior do estômago e das alças intestinais, o contraste demonstrou ser muito eficiente, uma vez que 10 dos 15 pacientes tiveram melhor visualização destas estruturas anatômicas. É importante salien- tar três aspectos dos nossos resultados. $\mathrm{O}$ primeiro deles é que em todos os voluntários em que havia sobreposição do estômago ou duodeno, ela desapareceu após a utilização do meio de contraste. Esta melhora ganha importância quando lembramos que o hepatocolédoco é a estrutura mais longa da via biliar, sede da maioria das afecções que promovem obstrução.

Em segundo lugar, em apenas um paciente não houve melhora na visualização do ducto pancreático comum, provavelmente decorrente do fato de o voluntário só referir, após uma segunda indagação, que não havia feito o jejum solicitado, e pior, havia acabado de se alimentar momentos antes do exame.

$\mathrm{E}$, finalmente, no que se refere à visualização da papila, não pudemos comprovar, estatisticamente, a eficácia do meio de contraste, uma vez que poucos exames apresentavam sobreposição desta estrutura já na fase pré-contraste (4/15), não permitindo tratamento estatístico. Um estudo com maior casuística será necessário para esclarecer este dado.

O uso do meio de contraste oral negativo pode ser questionado por causa dos efeitos colaterais e da baixa tolerância, uma vez que para o diagnóstico utilizamos, muitas vezes, as imagens originais, prévias à reconstrução, para interpretação. Em contrapartida, a colangio-RM apresenta-se como alternativa à CPRE, pois os médicos solicitantes (geralmente gastroenterologistas) estão familiarizados com imagem em duas dimensões das vias biliares, semelhante àquela obtida por reconstruções com técnica de projeção de intensidade máxima na colangio-RM. Nestas imagens, a sobreposição de alças intestinais pode reduzir a confiabilidade diagnóstica. Para obtermos esta imagem, semelhante à CPRE e sem sobreposição de alças, torna-se quase obrigatório o preparo intestinal com o meio de contraste negativo.

Outro ponto importante a ser considerado é o volume de contraste administrado. A embalagem do Lumirem ${ }^{\circledR}$ é composta por três frascos com $300 \mathrm{ml}$ por unida- de, totalizando dose de $900 \mathrm{ml}$, preconizada pelo fabricante para estudos abdominais. No nosso estudo, utilizamos apenas $300 \mathrm{ml}$ e obtivemos resultados bastante satisfatórios na avaliação das vias biliares. Acreditamos, portanto, que para exames de colangio-RM, esta dose reduzida de contraste oral seja suficiente.

Concluindo, o meio de contraste oral negativo para colangio-RM melhora a visualização das vias biliar e pancreática, podendo ser utilizado sempre que necessário, em dose reduzida e a despeito dos seus brandos efeitos colaterais e tolerância discutível. É importante observar que, atualmente, estamos usando de rotina este contraste nos exames de colangio-RM, em virtude dos resultados obtidos.

\section{Agradecimento}

Ao engenheiro Eduardo Figueiredo, pela inestimável colaboração e suporte técnico.

\section{REFERÊNCIAS}

1. Barish MA, Soto JA, Yucel EK. Magnetic resonance cholangiopancreatography of the biliary ducts: techniques, clinical applications, and limitations. Top Magn Reson Imaging 1996;8:302-11.

2. Soto JA, Barish MA, Yucel EK, et al. Pancreatic duct: MR cholangiopancreatography with a threedimensional fast spin-echo technique. Radiology 1995;196:459-64.

3. Takahara T, Saeki M, Nosaka S, et al. The use of high concentration ferric ammonium citrate (FAC) solution as a negative bowel contrast agent: application in MR cholangiography. Nippon Igaku Hoshasen Gakkai Zasshi 1995;55:697-9.

4. Hahn P, Stark DD, Saini S, Lewis JM, Wittenberg J, Ferrucci JT. Ferrite particles for bowel contrast in MR imaging: design issues and feasibility studies. Radiology 1987;164:37-41.

5. Cappeliez O, Delhaye M, Devière J, et al. Chronic pancreatitis: evaluation of pancreatic exocrine function with MR pancreatography after secretin stimulation. Radiology 2000;215:358-64.

6. Lonnemark M, Hemmingsson A, Carlsten J Ericsson A, Holtz E, Klaveness J. Superparamagnetic particles as an MRI contrast agent for the gastrointestinal tract. Acta Radiol 1988;29:599-602.

7. Lonnemark M, Hemmingsson A, Bach-Gansmo T, et al. Effect of superparamagnetic particles as oral contrast medium at magnetic resonance imaging. A phase 1 clinical study. Acta Radiol 1989;30: 193-6.

8. Bach-Gansmo A, Dupas B, Gayet-Delacroix M, Lambrechts M. Abdominal MRI using a negative contrast agent. Br J Radiol 1993;66:420-5. 